\title{
FLUIDS WITH STATE-DEPENDENT ELECTRONIC STRUCTURE
}

\author{
$\operatorname{AUTHOR}(\mathrm{S})$ : \\ Hensel, F.
}

\section{CITATION:}

Hensel, F.. FLUIDS WITH STATE-DEPENDENT ELECTRONIC STRUCTURE. 物性研究 1994, 62(2): 347-351

\section{ISSUE DATE:}

1994-05-20

URL:

http://hdl.handle.net/2433/95317

RIGHT: 


\title{
FLUIDS WITH STATE-DEPENDENT ELECTRONIC STRUCTURE
}

\author{
F. Hensel \\ Institute of Physical Chemistry and Materials Science Center \\ Philipps University of Marburg \\ D-3550 Marburg, F.R.G.
}

Fluid metals are fundamentally distinct from normal insulating fluids such as argon in that their electronic and molecular structures are strongly depending on the thermodynamic state of the system. It is obvious that there are substantial changes in the nature of the bonding of metals upon evaporation, at which point there is a transition from a metallic bonding state to an insulating state. The liquid just above its melting point possesses a large degree of correlation in the atomic positions, and the small changes of most electronic properties on melting show that the electronic structure of the liquid is quite similar to that of the solid. Consequently, the liquid metallic phase is usually treated as a monoatomic state which typifies the solid structure. It is regarded as built up of single screened ions, each diffusively uncoupled from every other. For the alkali metal vapor, on the other hand, it is known from optical absorption- [1], magnetic susceptibility- [2], and equation of state data [3] that the dominant stable species are the neutral atom and covalently bonded dimer molecules.

Whilst the two limiting cases of the dense liquid metal near the melting point and the low density vapor phase are reasonably well understood, our knowledge of the connection between these two limits through the liquid-vapor critical and supercritical regions of the phase diagram lags far behind. Nearer to the critical point, the liquid density is much less and the vapor density much greater. Thus, while liquid alkali metals might be regarded as monoatomic near the melting point, the viewpoint might be rather different for the lower density liquid regime where dimers or higher clusters can be the appropriate submits. The dimer molecules present in the vapor might well survive the passage to the metallic liquid.

The inference of an appreciable concentration of dimer associations in the critical regions of alkali metals is consistent with the most accurate equation-of-state data for cesium [3], rubidium [3], and potassium [4]. The effect of association is vividly illustrated by the comparison of the experimentally determined critical compression factors $Z_{c}$ for $C s(0.204), R b$ 
$(0.219)$ and $\mathrm{K}(0.179)$ with that of the hard sphere plus mean field fluid (0.358).

Straightforward calculations using the theory of Gubbins et al. [5] for phase equilibria of as sociating molecules with one bonding site [4] suggest that the dimer association is about $31 \%$ for $\mathrm{Cs}, 28 \%$ for $\mathrm{Rb}$ and $34 \%$ for $\mathrm{K}$.

The problem that throughout the fluid range of an alkali metal the dynamic units can change significantly with density and temperature, i.e. that vestiges of dimers can persist in the lower-density range of the liquid, has stimulated neutron scattering measurements of the static structure factor $\mathrm{S}(\mathrm{Q})$ of $\mathrm{Cs}[6]$ and $\mathrm{Rb}$ [7]. The data obtained show that the dominant effect of thermal expansion of the liquid is a reduction of the average coordination number (thermal hole formation), while the average interparticle distance grows only very little. However, within standard approximations of theoretical descriptions and within experimental uncertainties, the $S(Q)$ data do not provide a clear-cut answer to the question mentioned above. The problem is that quite different views of the microscopic structure of expanded liquid alkali metals are possible to describe $\mathrm{S}(\mathrm{Q})$ reasonably well.

More interesting insight into the question whether remnants of the diatomic unit, present in the dense vapor phase of alkali metals, can survive the condensation to the liquid state can be obtained by examining recent coherent inelastic neutron scattering spectra for liquid $\mathrm{Rb}$ [8], [9] which extend nearly to the critical region. The critical data for $\mathrm{Rb}$ are: $\mathrm{T}_{\mathrm{C}}=2017$ $\mathrm{K}, \mathrm{p}_{\mathrm{c}}=124.5 \mathrm{bar}, \rho_{\mathrm{c}}=0.29 \mathrm{~g} / \mathrm{cm}^{3}$ [3]. From the early work of Copley and Rowe [10], [11] it is known that $\mathrm{Rb}$ near its melting point at $320 \mathrm{~K}$, unlike dense Lennard-Jones (inert gas) systems, exhibits distinct longitudinal collective excitations up to relatively high Q-values. The measured dispersion relation (shown in Figure 1) resembles very much that of longitudinal phonons in crystalline $\mathrm{Rb}$. The presence of such excitations for liquid $\mathrm{Rb}$, and their absence in inert gas liquids, has been attributed to the characteristic features of the effective metallic interaction potential. This view has been prompted by an investigation of liquid $\mathrm{Rb}$ near its melting point in a molecular dynamics calculation using a typical metallic interaction potential [12], [13]. The latter is in excellent agreement with the neutron scattering data.

The recent high-temperature experiments [8], [9] evidently show that these excitations are still visible at higher temperatures up to $1673 \mathrm{~K}$, but the widths of the peaks in $S(Q, \omega)$ are so large that they are no longer resolvable and only broad shoulders are indicated. The dispersions obtained from the maxima in the longitudinal current density correlation functions $\mathrm{J}_{1}(\mathrm{Q}, \omega)=\omega^{2} \cdot \mathrm{S}(\mathrm{Q}, \omega) / \mathrm{Q}^{2}$ (see Figure 2) are also shown in Figure 1. 
As noted above, the dominant effect of thermal expansion on the structure of fluid alkali metals is a reduction of the average coordination number rather than an increased near neighbor distance. The latter grows only very little. The consequences of these structure changes are reflected in Figure 1. The minima of the dispersion curves shift slightly to lower $Q$ values as the maxima of $S(Q)$ and the free particle dispersion becomes more pronounced at higher temperature (lower density) as one would expect. Interestingly, the accompanying molecular dynamics calculations [14], [15] using typical metallic interaction potentials are in good agreement with the experimental results up to $1673 \mathrm{~K}$ and a density of about three times the critical density which indicates that the mean forces given by the metallic binding and the screening by the nearly free electrons are still controlling the dynamics of liquid $\mathrm{Rb}$ at this conditions. This is no longer the case, if the temperature is incresed still further (see e.g. in Figure $2, T=1873 \mathrm{~K}$, the corresponding density is about twice the critical density), a clear change in the shape of $\mathrm{J}_{1}(\mathrm{Q}, \omega)$ is observed. $\mathrm{J}_{1}(\mathrm{Q}, \omega)$ at $\mathrm{Q}=1.3 \AA-1$ exhibits well defined excitation peaks around $h \omega$ about 3.2, 6.4, and 9.6 $\mathrm{meV}$. One way of interpreting this observation is to propose that the peak is due to an optic-type mode, i.e. optic vibration, in which two species tend to move in opposite directions. The intensities of such excitation peaks depend strongly on the momentum transfer $\mathrm{Q}$. This can be seen at a glance by considering the model for scattering from a particle executing harmonic oscillations about a center for $k_{B} T>h \omega_{0}$ :

$$
\begin{gathered}
S(Q, \omega)=\sum_{n=-\infty}^{\infty} I_{n}(y) e^{-y} \delta\left(\omega-n \omega_{0}\right) \\
y=\frac{h^{2} Q^{2} k_{B} T}{M\left(h \omega_{0}\right)^{2}}
\end{gathered}
$$

Herein $\operatorname{In}_{\mathrm{n}}(\mathrm{y})$ are modified Bessel-Functions of the first kind and $\mathrm{n}$-th order, $h \omega_{0}$ is the energy difference between adjacent energy-levels of the oscillating particle and the $\delta$-function under the sum ensures conservation of energy during the interaction between the neutron and the molecule. $\mathrm{k}_{\mathrm{B}}$ is the Boltzmann-constant and $\mathrm{M}$ the mass of the particle. In order to demonstrate that the experimentally observed variation of the observed scattering laws with momentum transfer $Q$ is qualitatively consistent with the predictions of the harmonic oscillator model we compare in Figure 2 the experimental and calculated longitudinal current correlation function $\mathrm{J}_{1}(\mathrm{Q}, \omega)=\omega^{2} \cdot \mathrm{S}(\mathrm{Q}, \omega) / \mathrm{Q}^{2}$ for the curve at $1873 \mathrm{~K}$. The shapes of the two curves are surprisingly similar. This observation is consistent with the view 
that paired units with intramolecular dynamic effects are present in liquid expanded $\mathrm{Rb}$ under these conditions.

\section{REFERENCES:}

[1] B. Knuth, Doctoral Thesis, University of Marburg, Germany, 1990

[2] W. Freyland, Phys. Rev. B20, pp. 5104-5110(1979)

[3] S. Jüngst, B. Knuth, and F. Hensel, Phys. Rev. Lett. 55, pp.2160-2163 (1985)

[4] G.-F. Hohl, Doctoral Thesis, University of Marburg, Germany, 1992

[5] G. Jackson, W. G. Chapman, and K. E. Gubbins, Mol. Phys. 65, pp. 1-31 (1988)

[6] R. Winter, T. Bodensteiner, W. Gläser, and F. Hensel, Ber. Bunsenges. Phys. Chem. 91, pp. 1327-1330 (1987)

[7] G. Franz, W. Freyland, W. Gläser. F. Hensel, and E. Schneider, "Structure of Expanded Liquid Rubidium by Neutron Diffraction", in Proceedings of the 4th International Conference of Liquid and Amorphous Metals, J. Phys. Collog. C8 41, pp. 194-198 (1980)

[8] W.-C. Pilgrim, R. Winter, F. Hensel, C. Morkel, and W. Gläser, Ber. Bunsenges. Phys. Chem. 95, pp. 1133-1136 (1991)

[9] W.-C. Pilgrim, Doctoral Thesis, University of Marburg, Germany, 1992

[10] J. R. D. Copley, and J. M. Rowe, Phys. Rev. Lett. 2, pp. 49-52 (1974)

[11] J. R. D. Copley, and J. M. Rowe, Phis. Rev. A9, pp. 1656-1666 (1974)

[12] A. Rahman, Phys. Rev. Lett. 32, pp. 52-54 (1974)

[13] A. Rahman, Phys. Rev. A9, pp. 1667-1671 (1974)

[14] K. Hoshino, H. Ugawa, and M. Watanabe, J. Phys. Soc. Japan 61, pp. 2182-2185 (1992)

[15] G. Kahl. S. Kambayashi, and G. Nowotny, J. Non-Cryst. Solids 15, pp. 156-158 (1993)

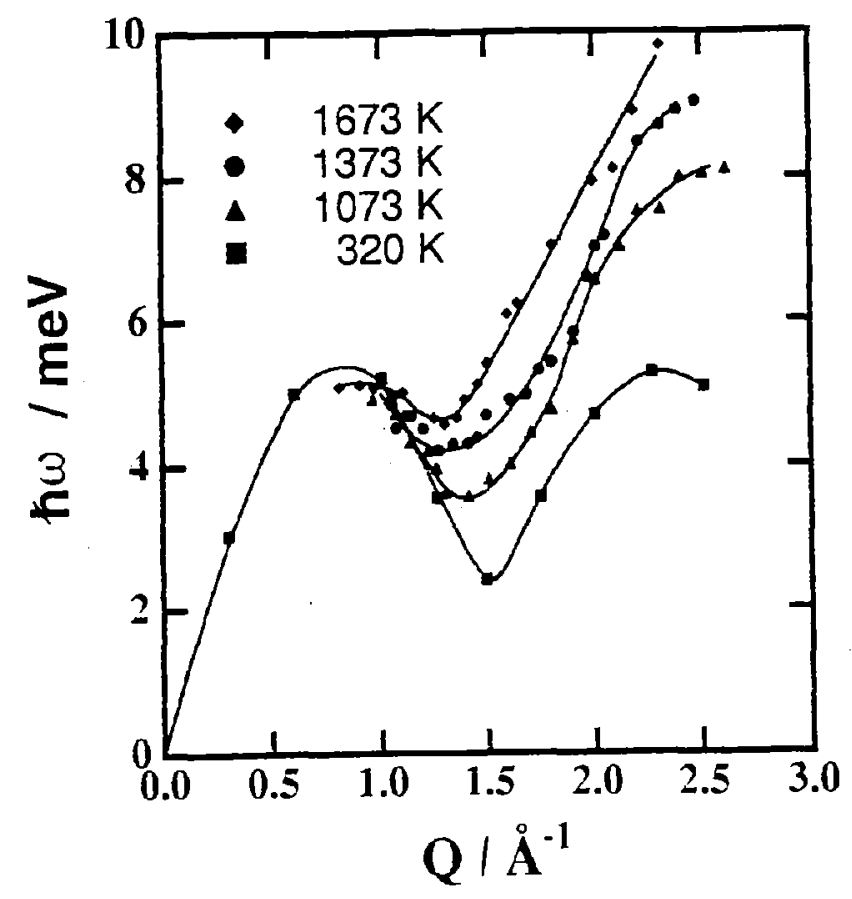

Figure 1. Dispersion curves obtained from the maxima in the longitudinal current comelation function for liquid $\mathrm{Rb}$ at conditions along the liquid-vapor coex istence line. The data at $320 \mathrm{~K}$ are from ref. $[9]$ and the high temperature data from ref. [10]. 


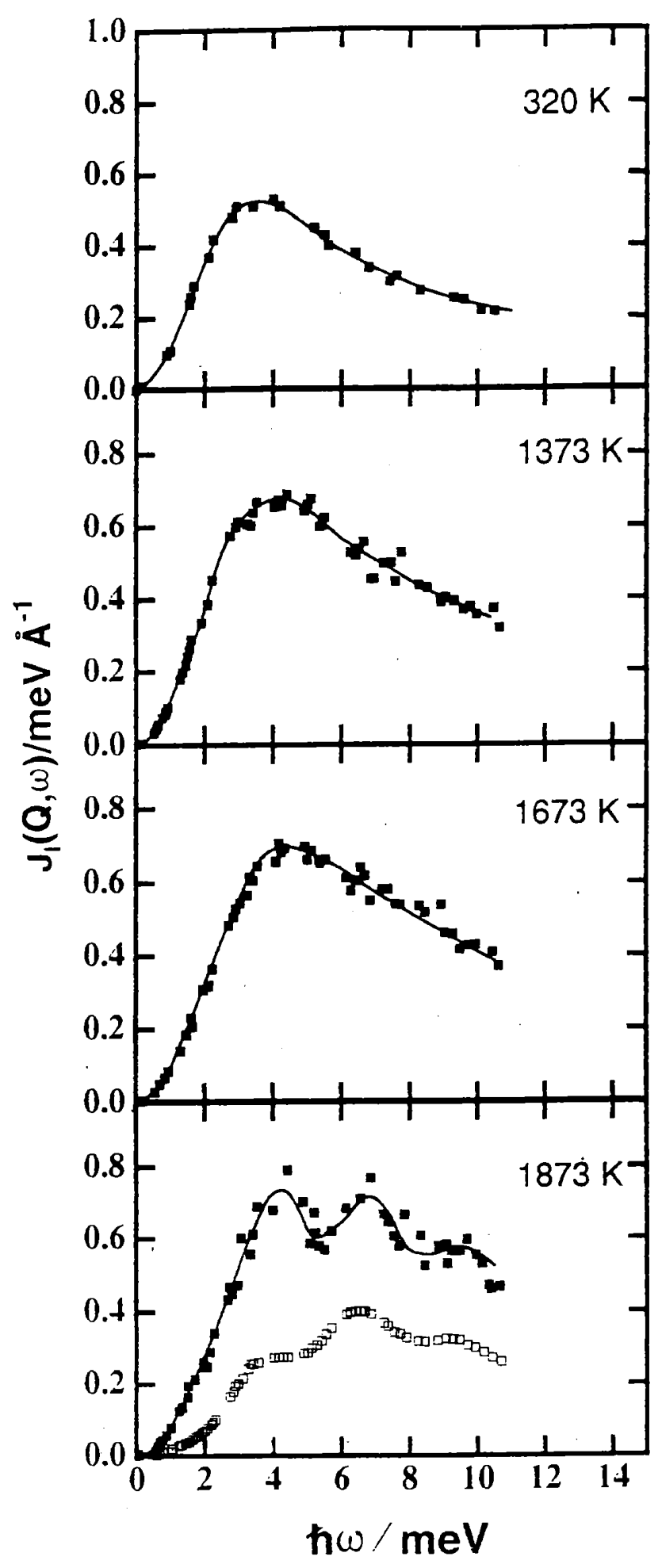

Figure 2. The experimental longitudinal current correlation functions of liquid $\mathrm{Rb}$ at a constant wavevector $\mathrm{Q}=1.3 \AA^{-1}$ for different temperatures along the liquid-vapor coexisting line. 